\title{
Roteamento de veículos sob restrições de capacidade e janelas de tempo em pequenas empresas utilizando as heurísticas das economias e da varredura
}

Routing of vehicles under capacity constraints and time windows in small enterprises using savings and sweep heuristics

\section{De rutas para vehículos bajo las limitaciones de capacidad $y$ ventanas de tiempo en las pequeñas empresas que utilizan heurísticas de ahorro y barrer}

WeLLINGTON GONÇALVES'

WilLIAN Sian HeRZOG"

Resumo A expansão das operações comerciais por meio do surgimento de mercados potenciais e do crescimento da globalização, tem direcionado os tomadores de decisão a maior atenção sobre a gestão das cadeias de distribuição. Ao selecionar um sistema de transporte, as empresas devem considerar o custo total de deslocamento, e não apenas o valor do frete, por conseguinte, torna-se importante a diminuição dos custos, que pode ser alcançada com a otimização do planejamento de rotas. A complexidade que envolve os processos de distribuição de cargas, bem como, o aumento do número de partes envolvidas, demandam soluções que auxiliem tomadas de decisão e, nesse contexto, estão inseridos os Problemas de Roteamento

Universidade Federal do Espírito Santo (UFES), São Mateus/ES - Brasil.

II Universidade Federal do Espírito Santo (UFES), São Mateus/ES - Brasil. 
de Veículos, (PRV) que, ao serem resolvidos, podem auxiliar na elaboração e determinação de rotas para uma frota de veículos, a partir de um depósito central ligado a um conjunto de clientes. O objetivo deste trabalho é a aplicação das heurísticas construtivas das Economias e da Varredura para resolução de um PRV sob restrições de capacidade e janelas de tempo. Os testes são ancorados em um estudo de caso, usando dados reais de uma pequena empresa do setor atacadista, no qual são consideradas sequências de locais a serem atendidos, obtidas pela remoção repetida de um vértice de sua rota atual e reinserção em outra rota. Por conseguinte, os resultados do uso de heurísticas construtivas para resolução do PRV sob restrições de capacidade e janelas de tempo mostraram-se adequados à realidade vivenciada pela pequena empresa, além de poder servir como ferramenta para a elaboração de estratégias competitivas. Palavras chave: Problema de Roteamento de Veículos. Heurísticas construtivas. PequeNA EMPRESA.

AвSTRACт The expansion of business operations through the emergence of potential markets and the growth of globalization has directed decision-makers to pay greater attention to the management of distribution chains. When selecting a transportation system companies should consider the total cost of travel, not just the freight value, therefore, it becomes important to reduce costs, which can be achieved by optimizing route planning. The complexity of the load distribution processes, as well as the increase in the number of involved parties, demand solutions that help decision making and, in this context, are inserted the Vehicle Routing Problems (VRP) that, when solved, can assist in the design and determination of routes for a fleet of vehicles, from a central depot connected to a set of customers. The objective of this work is the application of the constructive heuristics of the Savings and the Scan to solve a VRP under capacity constraints and time windows. The tests are based on a case study, using real data from a small company in the wholesale sector, in which, are considered sequences of places to be served, obtained by the repeated removal of a vertex of its current route and reinsertion in another route. Therefore, the results of the use of constructive heuristics to solve the VRP under capacity restrictions and time windows, were adequate to the reality experienced by the small company, besides being able to serve as a tool for the elaboration of competitive strategies.

Key-words: Vehicle Routing Problem. Constructive heuristic. Small Business.

REsumen La expansión de las operaciones comerciales a través de los mercados potenciales aparición y el crecimiento de la globalización, se ha dirigido a los tomadores de decisiones más atención en la gestión de cadenas de suministro. Al seleccionar un sistema de transporte de las empresas deben considerar el costo total del viaje, no sólo el valor de la carga, por lo tanto, es importante para reducir los costos, lo cual puede lograrse mediante la optimización de la planificación de rutas. La complejidad que rodea los procesos de distribución de carga, así como aumentar el número de partes involucradas, demandan soluciones que ayudan a la toma de decisiones y en este contexto se incluyen la Vehicle Routing Problem (VRP) que, cuando se resuelve, puede ayudar en la elaboración y determinación de rutas para una flota de vehículos desde un almacén central vinculada a un conjunto de clientes. El objetivo de este 
trabajo es la aplicación de Ahorros heurísticas constructivas y escanear para la resolución de un VRP en virtud de las limitaciones de capacidad y ventanas de tiempo. Las pruebas se basan en un estudio de caso, utilizando datos reales de una pequeña empresa en el sector mayorista, que se consideran que deben cumplir las secuencias de ubicaciones, obtenido por eliminación repetida de un vértice de la ruta actual y la reinserción en otra ruta. Por lo tanto, los resultados del uso de la heurística de la resolución constructiva de la VRP en virtud de las limitaciones de capacidad y ventanas de tiempo demostraron ser adecuada a la realidad que viven los pequeños negocios, y pueden servir como una herramienta para el desarrollo de estrategias competitivas. Palabras clave: Vehicle Routing Problem. Heurística constructiva. Pequeños Negocios.

\section{INTRODUÇÃO}

A expansão das operações comerciais por meio do surgimento de mercados potenciais e do crescimento da globalização tem direcionado os tomadores de decisão a terem maior atenção sobre a gestão das cadeias de distribuição. A complexidade que envolve os processos de distribuição, bem como, o aumento do número de partes envolvidas, indicam a necessidade de sistemas que possam apoiar com maior eficiência as tomadas de decisão.

O setor atacadista brasileiro insere-se nesse contexto, podendo ser visto como uma importante ligação entre a indústria e o mercado varejista. Por exemplo, no ano de 2013 o setor obteve um crescimento de 4,4\% em relação ao ano de 2012, com um faturamento de USD 64,27 bilhões de dólares, sendo a região sudeste responsável por quase $50 \%$ do faturamento anual brasileiro (ABAD, 2015).

As sazonalidades causadas pelas condições das vias de tráfego e da mobilidade urbana têm levado ao crescimento da demanda e requerido das empresas que atuam com distribuição de cargas, soluções que ampliem suas alternativas e otimizem o tempo de atendimento.

Ao selecionar um sistema de transporte, as empresas precisam considerar o custo total de deslocamento, e não apenas o valor do frete, por conseguinte, torna-se importante a diminuição dos custos, que pode ser alcançada com a otimização do planejamento de rotas que deve iniciar e terminar no depósito (RIBEIRO; LAPORTE, 2012). Deve-se ainda observar que a demanda em qualquer rota estabelecida não exceda a capacidade de atendimento do veículo, e que cada ponto seja visitado pelo menos uma vez (BALDACCI; MINGOZZI; ROBERTI, 2012).

Tais elementos caracterizam o problema de um depósito para vários veículos, podendo ser entendido como um Problema de Roteamento de Veículos (PRV) clássico, que possui o objetivo de encontrar um conjunto de rotas de entrega que partem de um único Centro de Distribuição (CD), e ao terminar as entregas nos pontos de demanda, retornam ao mesmo ponto de partida, minimizando o custo total (LAPORTE et al., 2000; LOCOMME et al., 2015; GOODSON, 2015).

O PRV tem sido utilizado na literatura para projetar uma rota ideal para uma frota de veículos que tem por objetivo atender a um conjunto de clientes, segundo um conjunto de restrições, sendo também empregado no planejamento da entrega e coleta das cargas, servindo de ferramenta para o planejamento da gestão da cadeia de distribuição. Devido às necessi- 
dades de agilização da informação, ao aumento do uso computacional e da utilização de heurísticas e metaheurísticas, esses problemas tendem a ser resolvidos de forma integrada com a simulação de cenários. As empresas têm empregado softwares específicos como Route, TransCAD, Truckstops, Roadshow, RouteSmart etc., que empregam métodos como das Economias, Vizinho mais próximo, Varredura, entre outros, para planejar soluções de roteamento com a finalidade de acompanhar o desenvolvimento e melhorar a eficiência de suas atividades (LIN, 2014). Neste trabalho, optou-se por utilizar uma metodologia de pesquisa que emprega abordagem manual de heurísticas, que pode ser adaptável a diversos cenários, essa escolha foi realizada devido aos investimentos serem inferiores à aquisição desses softwares, tornando-a acessível à pequenas empresas.

O método das economias ou Savings Algorithm (SA), de Clarke e Wright (CLARKE; WRIGHT, 1964), e o método da varredura ou Sweep Algorithm (SWA), de Gillett e Miller (1964) estão entre as heurísticas de construção mais utilizadas para o PRV (POLAT et al., 2015; DAAMEN; PHILLIPSON, 2015; RODRÍGUEZ; RUIZ, 2012). O SA tem sido aplicado em situações que envolvem a movimentação de cargas em meios urbanos, podendo auxiliar no dimensionamento dos tempos de rota, e no gerenciamento da utilização da capacidade máxima dos veículos, tem a propriedade de inserir restrições e limitações, além de oferecer condições para minimizar a distância total percorrida (POLAT et al., 2015; DAAMEN; PHILLIPSON, 2015). O SWA tem sido empregado na literatura para a resolução do PRV e seus variantes, devido à sua aplicação ser direcionada a reunir um grupo de paradas em uma rota de acordo com o ângulo polar entre as paradas e o depósito (RODRÍGUEZ; RUIZ, 2012).

O presente trabalho busca propor alternativas para um PRV especificamente direcionado a pequenas empresas, que pode ser modelado como um PRV de coleta e entrega de cargas, com restrições de janelas de tempo e de atendimento junto ao cliente. Apresentar uma proposta para a resolução do PRV por meio de heurísticas construtivas (SA e SWA), utilizando informações sobre a demanda de movimentação de cargas de uma pequena empresa do setor atacadista, atuante nas microrregiões Central Serrana, Centro-Oeste, Metropolitana, Nordeste, Noroeste e Rio Doce do Estado do Espírito Santo (Brasil), é o objetivo deste trabalho.

\section{MATERIAIS E MÉTOdOS}

Para compor a validação deste trabalho foi utilizada uma amostra não-probabilística, selecionada por conveniência, sendo realizado um estudo de caso em uma pequena empresa do setor atacadista situada no município de Linhares - Estado do Espírito Santo (Brasil). Segundo as rotinas de trabalho, operação e gerenciamento de atividades, foram disponibilizados pela empresa os dados correspondentes ao processo de movimentação de cargas (coleta e entrega), a localização e caracterização do depósito e da demanda, as restrições de horário de atendimento do depósito e dos clientes, os tipos e capacidades dos veículos disponíveis. As heurísticas construtivas SA e SWA foram selecionadas para a implementação do PRV por fornecerem soluções de qualidade aceitável, implementação e compreensão simplificada, que visam encontrar rotas que possam auxiliar os tomadores de decisão na minimização dos custos. 
O depósito é considerado ponto de origem e também o ponto final do arranjo da roteirização. Os dados da demanda, restrições e janelas de tempo foram obtidos por meio de entrevistas estruturadas com o gerente de operações e administrativo da empresa pesquisada por meio de uma pesquisa survey. Para obter as distâncias entre os pontos a serem atendidos, assim como dos mesmos até o depósito, utilizou-se o Google Earth® software (GOOGLE INC, 2015), adotando-se um fator de ajuste das distâncias Euclidianas (distâncias métricas) de 1,23 obtido por meio da média entre as distâncias dos estabelecimentos a serem atendidos (GONÇALVES et al., 2014). A partir da literatura (CEPOLINA; FARINA, 2015), em observações de campo e nas condições relatadas pelos gerentes da empresa, considerou-se um tempo médio de parada igual a 10 minutos, independente da demanda, e um tempo médio de carregamento e descarregamento igual a 2 minutos por unidade, outra característica relatada foi que semanalmente os veículos entregam e recolhem produtos, iniciando as rotas a partir do depósito, em que os compartimentos de cada veículo são preenchidos de acordo com os pedidos e programações previamente demandadas. A partir dessas informações e das observações in loco, e do consenso obtido junto aos gerentes, estimou-se a produção mensal de entrega e coleta em cada cliente. Por conseguinte, para a elaboração da proposta de solução do PRV utilizou uma planilha eletrônica para condensar e analisar os resultados obtidos por meio das heurísticas construtivas SA e SWA.

A Figura 1 ilustra a rotina utilizada para empregar a heurística SWA, a qual foi feita em duas fases (estruturação e aplicação). A obtenção dos dados foi concretizada por meio do Google Earth®, sendo em seguida alocados todos os pontos de entrega e coleta no mapa georreferenciado obtido. A seguir, tendo por base essa alocação, foi realizada a aplicação do SWA.

Figura 1 - Obtenção dos roteiros pela SWA

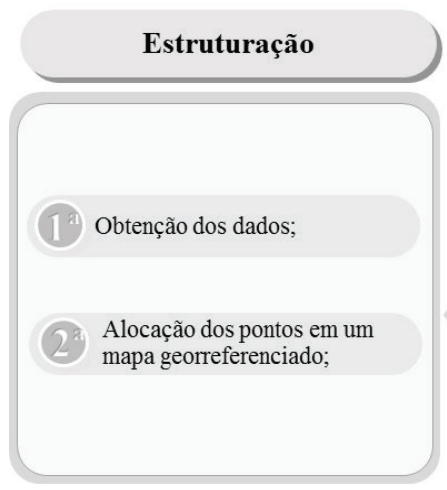

\section{Aplicação}

Tomando o depósito como centro, definir um eixo passando por ele. Este eixo geralmente coincide com a linha horizontal; Girar o eixo em torno do $\mathrm{CD}$ no sentido anti-horário até que inclua um cliente;

Testar o cliente em potencial, verificando se o mesmo pode ser incluído no roteiro em formação, por meio da análise das restrições de tempo, capacidade e horários existentes;

6. Se o novo cliente não puder ser incluído no roteiro em formação, é sinal que as possibilidades desse roteiro se esgotaram. Nesse caso, é iniciada uma nova rota. O processo termina quando todos os clientes tiverem sido incluídos num roteiro. Por fim, é realizado a análise de sensibilidade.

Na sequência, a Figura 2 delineia a forma de emprego da heurística SA, sendo a mesma concretizada em duas fases (estruturação e aplicação). A obtenção dos dados foi feita por meio do Google Earth®, sendo em seguida calculadas as distâncias e possíveis ganhos com roteamentos. Todos esses passos foram realizados até que todas as cidades a serem atendidas fossem inseridas em um roteiro. A seguir, considerando uma variação de janela de tempo de 8 horas para 10 horas, foi realizada a verificação do comportamento do custo total (análise de sensibilidade). 
Figura 2 - Rotina dos roteiros estabelecidos pela SA
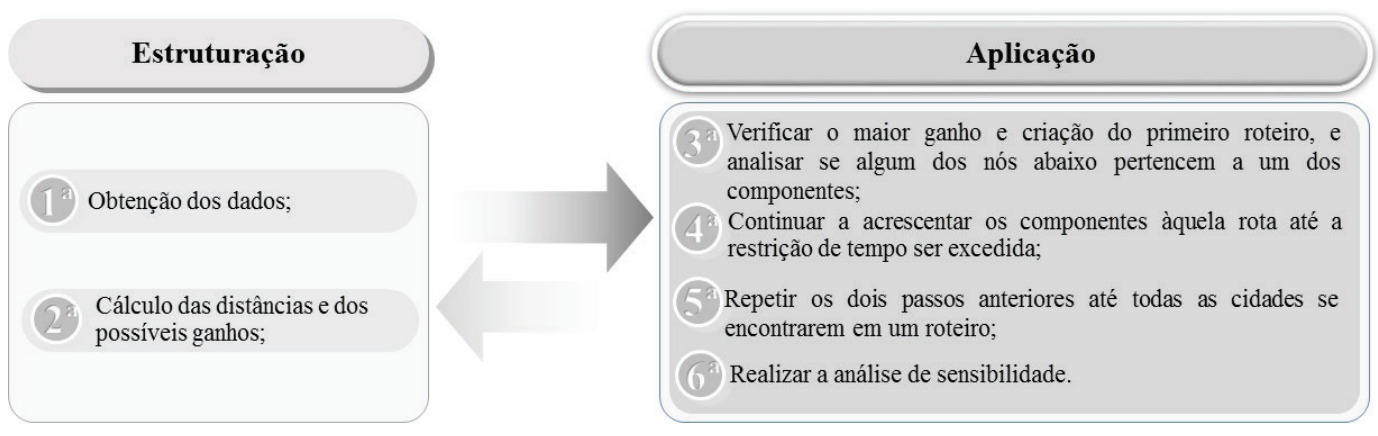

Para melhor entendimento dos fluxos de movimentação de cargas, foram realizadas cinco visitas in loco à empresa e às rotas ora praticadas, sendo ainda considerada para o cálculo do tempo de deslocamento a velocidade de $40 \mathrm{~km} / \mathrm{h}$, além de observações sob os percursos reais das rotas, que são realizadas em perímetro urbano (WILHEIM, 2013), sendo avaliada a frota disponível na empresa (1 [um] veículo urbano de carga com capacidade para até 3 toneladas e 2 [dois] caminhões toco ou semipesado com capacidade para até 6 toneladas) que realiza as entregas e coletas nas microrregiões Central Serrana (municípios de Itaguaçu, Itarana, Santa Maria de Jetibá e Santa Teresa), Centro-Oeste (municípios de Baixo Guandu, Colatina, Governador Lindenberg, Marilândia, São Gabriel da Palha e Vila Valério), Metropolitana (municípios de Cariacica, Fundão e Vila Velha), Nordeste (municípios de Conceição da Barra, Jaguaré, Montanha, Mucurici, Pedro Canário, Pinheiros, Ponto Belo e São Mateus), Noroeste (municípios de Água Doce do Norte, Águia Branca, Barra de São Francisco, Ecoporanga, Nova Venécia e Vila Pavão) e Rio Doce (municípios de Aracruz, Ibiraçu, João Neiva, Linhares, Rio Bananal e Sooretama). A Figura 3 ilustra geograficamente essas microrregiões.

As organizações necessitam rever constantemente seus Custos fixos $\left(C_{f}\right)$ e variáveis $\left(C_{v}\right)$, devido ao preço do produto final ser determinado pelo comportamento do mercado, e com isso, as variações de cenários mercadológicos influenciam diretamente o custo do transporte, assim como, o preço do produto para o cliente final (SOUZA et al., 2014). O cálculo do Custo da rota (C_rota) foi realizado por meio de uma formulação híbrida da literatura (ARAÚJO; BANDEIRA; CAMPOS, 2014; FERRARI, 2015), Equação 1, sendo considerados os $\left(C_{f}\right)$ e $\left(C_{v}\right)$ contidos na Tabela 1 , os quais foram adequados à realidade e necessidades da empresa, obtidos a partir das distâncias médias percorridas mensalmente (em km).

$$
C_{-} \text {rota }=D *\left(C_{v}+C_{f}\right)+P
$$

Em que:

D: Distância percorrida em quilômetros. [km]

P: Pedágio. [USD]

Devido às janelas de tempo influenciarem diretamente os custos totais, tomando por base os dados históricos e informações gerenciais da empresa estudada, as heurísticas SWA e SA foram empregadas considerando dois intervalos de tempo (8 horas e 10 horas), tornando 
possível a realização de uma análise de sensibilidade do comportamento dessas janelas no custo total.

Figura 3 - Demonstração das Microrregiões atendidas

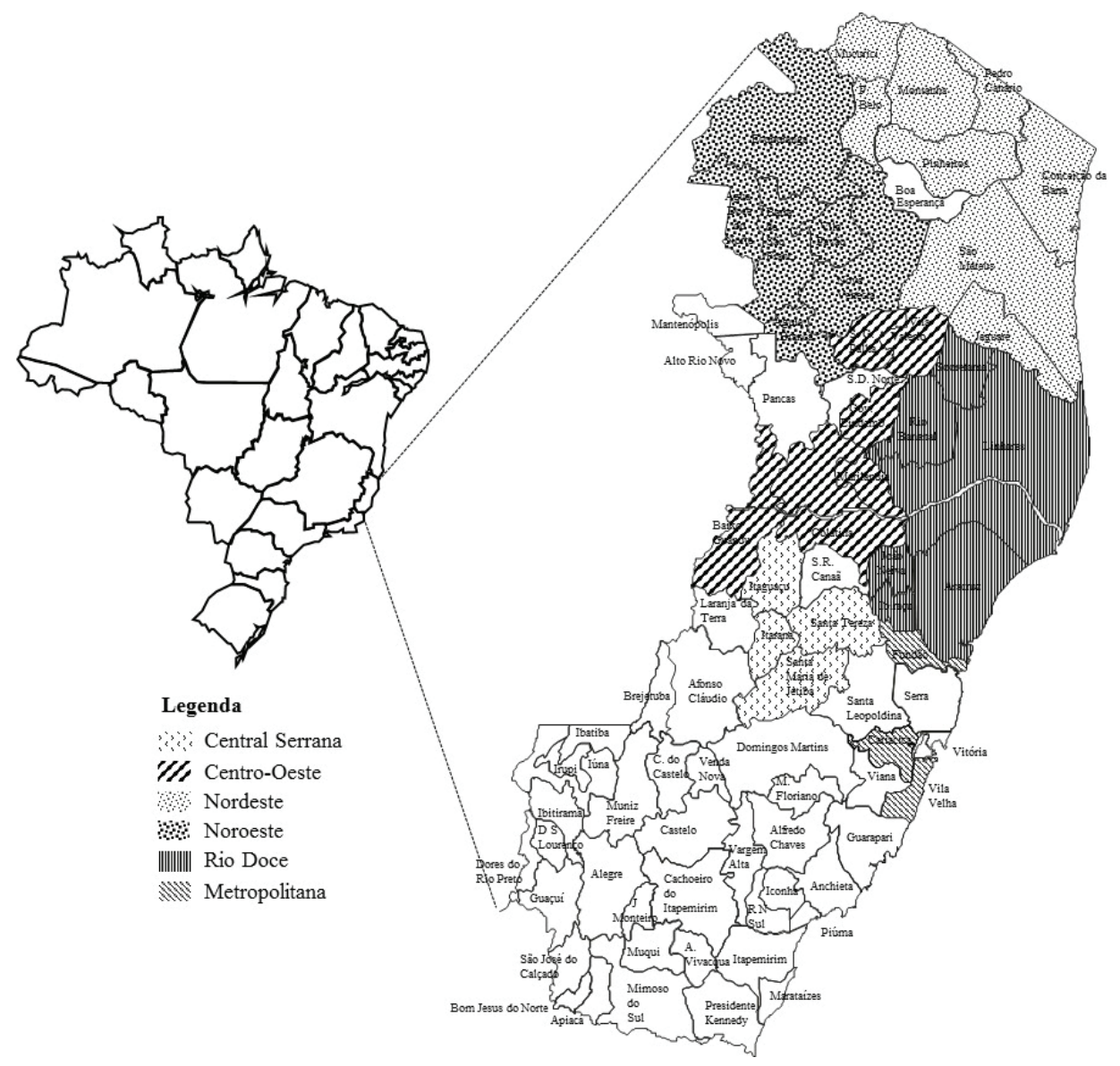

Tabela 1 - Custos fixos e variáveis considerados no custo da rota

\begin{tabular}{cc}
\hline Custo & Descrição \\
\hline Fixo & Depreciação \\
& Custos Administrativos \\
& IPVA e Seguro obrigatório \\
& Manutenção \\
& Salários \\
Variáveis & Pneu \\
& Combustível \\
& Hora extra \\
\hline
\end{tabular}




\section{AplicaÇÃo e discussão dos Resultados}

A primeira heurística aplicada foi a SWA (Figura 1), iniciando-se com a realização de um diagnóstico para a obtenção dos dados referentes aos e (Tabela 2), e das microrregiões atendidas. Logo após, efetuou-se a alocação dos pontos a serem atendidos em um mapa georreferenciado com a finalidade de iniciar a aplicação do método (GOOGLE INC, 2015).

Tabela 2 - Custos considerados para o cálculo da rota

\begin{tabular}{lc}
\hline \multicolumn{1}{c}{ Descrição (Custo por km) } & Valor (USD) \\
\hline Depreciação & 0,017 \\
Custos Administrativos & 0,029 \\
IPVA e Seguro obrigatório & 0,011 \\
Manutenção & 0,004 \\
Salários & 0,040 \\
Pneu & 0,021 \\
Hora extra & 0,019 \\
Combustível & 0,169 \\
\hline
\end{tabular}

Em seguida, iniciou-se a fase de aplicação da heurística girando o eixo traçado no mapa, contornando o mesmo no sentido anti-horário até que se englobasse um município, foi repetido o passo anterior até que alguma restrição encerrasse qualquer continuidade. Após a criação da primeira rota, o processo foi reiniciado até que todos os municípios se encontrassem em um roteiro, por fim, foi verificada a existência de postos de pedágio, e em caso afirmativo esses foram adicionados ao custo do roteiro. Na aplicação inicial do SWA, foi obtido um total de 8 (oito) rotas (Figura 3), com um percurso total de $3.099 \mathrm{~km}$, tempo total de 62 horas e custo total da rota de USD 1.005,85, sendo possível verificar que a distância relativa de alguns municípios influencia diretamente nos resultados.

Posteriormente, ao ser realizada uma nova aplicação do SWA, em que foi alterado o fator restritivo de horas de 8 para 10, foi possível obter 7 (sete) rotas (Tabela 3) a partir desse cenário, com uma distância percorrida de $2.322 \mathrm{~km}$, tempo de $47 \mathrm{~h}$ e custo total de USD 786,93, com essa ação houve a redução dos custos em $21,77 \%$, representando uma economia de USD 218,92. A heurística SA (Figura 4) foi iniciada a partir da obtenção dos dados na aplicação anterior, a seguir houve a realização do cálculo das distâncias entre os municípios e dos ganhos para cada par de nó, utilizando o Google Earth ${ }^{\circledR}$ software (GOOGLE INC, 2015) e uma planilha eletrônica, sendo realizada a ordenação dos pares para iniciar a fase de aplicação da heurística, com isso, um roteiro para cada par foi criado, verificando-se o par com o maior ganho, e checando se os pares abaixo deste possuem algum ponto do par analisado, com o intuito de inserir esse nó à rota, desde que não ultrapassada a restrição de horas. 
Figura 3 - Demonstração dos roteiros estabelecidos

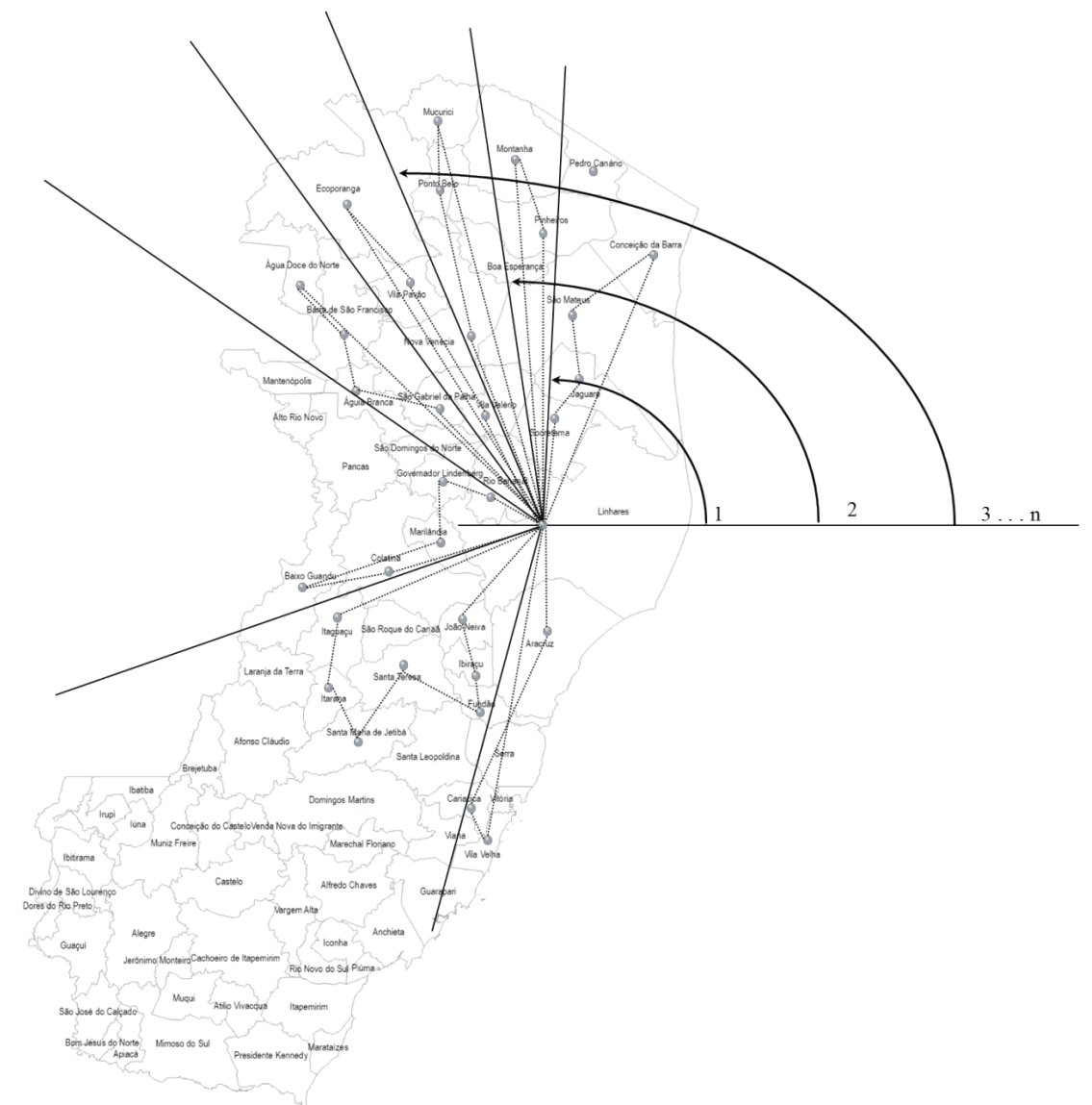

Tabela 3 - Rotas criadas pelo método de SWA com a ampliação do fator restritivo

\begin{tabular}{|c|c|c|c|c|c|}
\hline Rotas & 1 & 2 & 3 & 4 & 5 \\
\hline \multirow{8}{*}{ 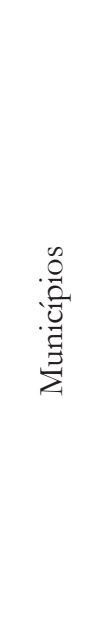 } & Sooretama & Vila Valério & Ecoporanga & Rio Bananal & Aracruz \\
\hline & Jaguaré & $\begin{array}{l}\text { Nova Ve- } \\
\text { nécia }\end{array}$ & $\begin{array}{l}\text { Água Doce do } \\
\text { Norte }\end{array}$ & Colatina & Fundão \\
\hline & São Mateus & Vila Pavão & $\begin{array}{l}\text { Barra de São } \\
\text { Francisco }\end{array}$ & Marilândia & Cariacica \\
\hline & $\begin{array}{c}\text { Conceição da } \\
\text { Barra }\end{array}$ & Ponto Belo & $\begin{array}{c}\text { São Gabriel da } \\
\text { Palha }\end{array}$ & Baixo Guandu & Vila Velha \\
\hline & Pedro Canário & Mucurici & $\begin{array}{c}\text { Governador Lin- } \\
\text { denberg }\end{array}$ & Itaguaçu & Ibiraçu \\
\hline & Montanha & & Rio Bananal & Itarana & João Neiva \\
\hline & \multirow[t]{2}{*}{ Pinheiros } & & & $\begin{array}{c}\text { Santa Maria de } \\
\text { Jetibá }\end{array}$ & \\
\hline & & & & Santa Teresa & \\
\hline
\end{tabular}


No que diz respeito à continuidade da aplicação do SA, houve a repetição da etapa anterior até que todos os municípios estivessem em uma rota, observou-se a presença de pontos de pedágios em alguns trajetos, os quais foram acrescentados aos custos da rota, com isso, nessa aplicação foram obtidos um total de 7 (sete) rotas, com uma distância percorrida de $2.862 \mathrm{~km}$, tempo total de 54 horas e um custo de USD 892,78.

A partir de uma alteração no fator restritivo de 8 para 10 horas, repetiu-se a aplicação da heurística, sendo obtido um total de 5 (cinco) rotas, com uma distância percorrida de 2.123 $\mathrm{km}$, tempo total de 44 horas e custo de USD 716,68, com isso, ao realizar uma comparação entre os custos percebeu-se que houve uma redução de $19,72 \%$, o que totaliza um valor de USD 176,10 .

Ao realizar as aplicações e as análises de sensibilidade do SWA e do SA foi possível visualizar que os resultados obtidos por meio do SA tendem oferecer melhores ganhos sob as perspectivas analisadas (Tabela 4), ao realizar uma comparação entre os dados obtidos (Tabela 4 e Tabela 5) e a solução empírica adotada na pequena empresa (empregando dados históricos), levando-se em conta os custos totais, percebe-se que o SA obteve um custo menor de 11,24\% para 8 horas e $8,93 \%$ para 10 horas em relação ao SWA.

Tabela 4 - Roteiros criados pelo SA com a ampliação do fator restritivo

\begin{tabular}{|c|c|c|c|c|c|}
\hline Rotas & 1 & 2 & 3 & 4 & 5 \\
\hline \multirow{8}{*}{ 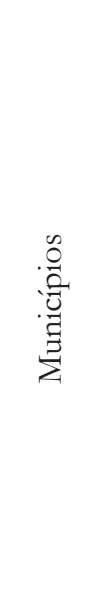 } & Pinheiros & $\begin{array}{c}\text { Barra de São } \\
\text { Francisco }\end{array}$ & $\begin{array}{c}\text { Santa Maria de } \\
\text { Jetibá }\end{array}$ & Vila Valério & Rio Banana \\
\hline & Montanha & $\begin{array}{c}\text { Água doce do } \\
\text { Norte }\end{array}$ & Santa Teresa & $\begin{array}{c}\text { São Gabriel da } \\
\text { Palha }\end{array}$ & $\begin{array}{l}\text { Governado } \\
\text { Lindenberg }\end{array}$ \\
\hline & Ponto Belo & Ecoporanga & Vila Velha & Itarana & Marilândia \\
\hline & Mucurici & Vila Pavão & Cariacica & Itaguaçu & \\
\hline & Pedro Canário & Nova Venécia & Fundão & Baixo Guandu & \\
\hline & $\begin{array}{c}\text { Conceição da } \\
\text { Barra }\end{array}$ & Jaguaré & João Neiva & Colatina & \\
\hline & São Mateus & Sooretama & Ibiraçu & Águia Branca & \\
\hline & & & Aracruz & & \\
\hline
\end{tabular}

Os resultados alcançados neste trabalho, ao serem comparados com dados históricos da pequena empresa, sugerem que as decisões operacionais podem ser aprimoradas com a metodologia proposta, tendo condições de maximizar resultados financeiros, assim como, permitir a oferta de uma ampliação do mix de serviços de distribuição e percepção de valor dos clientes. 


\section{CONSIDERAÇÕES FINAIS}

A aplicação de heurísticas construtivas na formulação de rotas pode contribuir com a definição de roteiros que atendam tanto aos anseios da demanda, quanto aos objetivos das empresas por meio da minimização dos custos.

A principal contribuição do presente trabalho está na proposição de uma metodologia conceitual que inclui características do PRV com restrições de capacidade e janelas de tempo, a fim de permitir que ela seja adaptável a diferentes realidades, sendo capaz de ser aplicada em instâncias práticas de pequenas empresas. Tal contexto enseja o desenvolvimento de abordagens proativas de roteamento de veículos, as quais abrangem a aplicação das heurísticas construtivas das Economias e da Varredura, possibilitando obter um tempo de resposta alinhado às necessidades de clientes e da empresa.

O objetivo deste trabalho foi atingido com a formulação da metodologia conceitual e da aplicação das heurísticas construtivas das Economias e da Varredura. Mesmo com resultados que podem ser avaliados como preliminares, este trabalho forneceu evidências que podem ser utilizadas para apoiar planejamentos e ações de distribuição de cargas.

A empresa objeto de estudo elaborava suas rotas de forma empírica, sem uma definição técnica ou estratégia específica para minimização dos seus custos, dessa forma, os pedidos eram recebidos e na sequência elaborados os percursos, caracterizando a ausência de uma programação prévia. Após a apresentação e utilização dessa proposta, a pequena empresa pôde definir roteiros baseados em seus dados históricos, passando a contar com uma ferramenta gerencial, a qual lhe permitiu planejar estratégias competitivas para cada região de atuação.

No caso estudado, o método SA foi o que obteve os melhores resultados com o menor custo total para os cenários propostos, em comparação com o SWA. O PRV pode ser resolvido e adaptado a realidades diversas, por meio de heurísticas e algoritmos que atendam às expectativas e ansiedades das empresas, e dessa forma, tem-se melhores condições para otimizar a eficiência da organização, além de promover um melhor atendimento aos seus clientes. Argumenta-se que as abordagens adotadas conseguiram apresentar uma proposta para a resolução do PRV por intermédio de heurísticas construtivas (SA e SWA), sendo validada por meio do estudo de caso realizado.

\section{REFERÊNCIAS}

ABAD - Associação Brasileira de Atacadistas e Distribuidores. Dados do setor. Disponível em: <http://www.abad.com.br>. Acesso em: 8 de março de 2015.

ARAÚJO, M. P. S.; BANDEIRA, R. A. M.; CAMPOS, V. B. G. Costs and freights charged in road cargo transportation: a comparative analysis between owner operators and companies. Journal of Transport Literature, v. 8, n. 4, p. 187-226, 2014.

BALDACCI, R.; MINGOZZI, A.; ROBERTI, R. Recent exact algorithms for solving the vehicle routing problem under capacity and time window constraints. European Journal of Operational Research, v. 218, n. 1, p. 1-6, 2012. 
CEPOLINA, E. M.; FARINA, A. A new urban freight distribution scheme and an optimization methodology for reducing its overall cost. European Transport Research Review, v. 7, n. 1, p. 1-14, 2015.

CLARKE, G. U.; WRIGHT, J. W. Scheduling of vehicles from a central depot to a number of delivery points. Operations research, v. 12, n. 4, p. 568-581, 1964.

DAAMEN, R.; PHILLIPSON, F. Comparison of heuristic methods for the design of edge disjoint circuits. Computer Communications, v. 61, p. 90-102, 2015.

FERRARI, P. Dynamic cost functions and freight transport modal split evolution. Transportation Research Part E: Logistics and Transportation Review, v. 77, p. 115-134, 2015.

GILLET'T, B. E.; MILLER, L. R. A heuristic algorithm for the vehicle-dispatch problem. Operations research, v. 22, n. 2, p. 340-349, 1974.

GONÇALVES, D. N. S.; GONÇALVES, C. M.; ASSIS, T. F.; SILVA, M. A. Analysis of the Difference between the Euclidean Distance and the Actual Road Distance in Brazil. Transportation Research Procedia, v. 3, p. 876-885, 2014.

GOODSON, J. C. A priori policy evaluation and cyclic-order-based simulated annealing for the multi-compartment vehicle routing problem with stochastic demands. European Journal of Operational Research, v. 241, n. 2, p. 361-369, 2015.

GOOGLE INC. Company Information. Google Earth®. Disponível em: < http://www.google. com.br/intl/pt-BR/earth>. Acesso em: 24 de maio de 2015.

LACOMME, P.; PRINS, C.; PRODHON, C.; REN, L. A multi-start split based path relinking (MSSPR) approach for the vehicle routing problem with route balancing. Engineering Applications of Artificial Intelligence, v. 38, p. 237-251, 2015.

LAPORTE, G.; GENDREAU, M.; POTVIN, J-Y.; SEMET, F. Classical and modern heuristics for the vehicle routing problem. International transactions in operational research, v. 7 , n. 4-5, p. 285-300, 2000.

LIN, C.; CHOY, K. L.; HO, G. T. S.; CHUNG, S. H.; LAM, H. Y. Survey of green vehicle routing problem: past and future trends. Expert Systems with Applications, v. 41, n. 4, p. 1.118-1.138, 2014.

POLAT, O.; KALAYCI, C. B.; KULAK, O.; GÜNTHER, H. O. A perturbation based variable neighborhood search heuristic for solving the Vehicle Routing Problem with Simultaneous Pickup and Delivery with Time Limit. European Journal of Operational Research, v. 242, n. 2, p. 369-382, 2015.

RIBEIRO, G. M.; LAPORTE, G. An adaptive large neighborhood search heuristic for the cumulative capacitated vehicle routing problem. Computers \& Operations Research, v. 39, n. 3, p. 728-735, 2012. 
RODRÍGUEZ, A.; RUIZ, R. A study on the effect of the asymmetry on real capacitated vehicle routing problems. Computers \& Operations Research, v. 39, n. 9, p. 2.142-2.151, 2012. SOUZA, M. A.; LEMOS, L. B.; ZORZO, L. S. Comércio Tradicional Versus Comércio Eletrônico: Um Estudo de Caso Sob o Olhar da Gestão dos Custos Logísticos. Sociedade, Contabilidade e Gestão, v. 9, n. 2, p. 80-100, 2014.

WILHEIM, J. Mobilidade urbana: um desafio paulistano. Estudos Avançados, v. 27, n. 79, p. 7-26, 2013.

Submetido em: 7-12-2016

Aceito em: 9-2-2017 